\title{
The Determination of Minimum Search Region Based on the Force Analysis after an Air Crash
}

\author{
Ran Li \\ North China Electric Power University Baoding \\ email: 1720304594@qq.com
}

Keywords: Search Region, Force Analysis, Air Crash

\begin{abstract}
In order to find the plane quickly, we demand to build a generic mathematical model to assist planning a search method to find the plane crashed in open water efficiently. What's more, in our model we shall consider the different types of accident plane and search plane based on the use of different electronics or sensors. Then we conclude that the core problem is to determine a reasonable search region. So we consult literature to get related knowledge. We build a model to determine the falling process and the minimum search region.
\end{abstract}

\section{Introduction}

For the first, we focus on determining the object search area. We divide the whole motor process into three parts according to the pre-set time axis [1]. More specifically, from $t_{0}$ to $t_{1}$ the plane keeps a uniform motion in a straight line along the original heading with a fixed height while from $t_{1}$ to $t_{2}$, due to the failure of engine, it glides without power until falls into the sea. And then, from $t_{2}$ to $t_{3}$, it will sink and won't stay still until reach the ocean floor. The timer shaft is as Figure 1 . To each definite $t_{1}$, it will correspond to a definite circuit region whose radius is $R_{1}$ in view of the indeterminancy of the gliding direction. Furthermore, as the change of $t_{1}$, the corresponding circuit region will do translation motion and gradually form a swath. Meanwhile, in order to ensure a comprehensive search area, we make a circle whose radius is $R_{2}$ in the boundary of the swath. The entire process is as Figure 2.

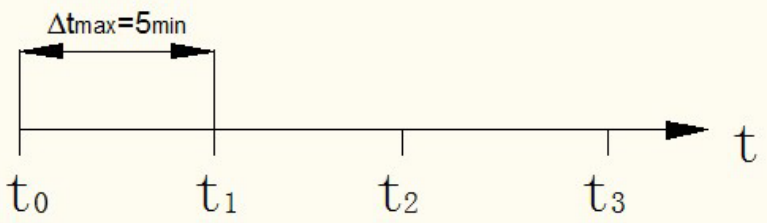

Figure 1 The timer shaft of the theoretical curves

(1)

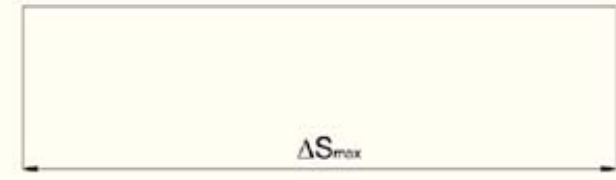

(2)

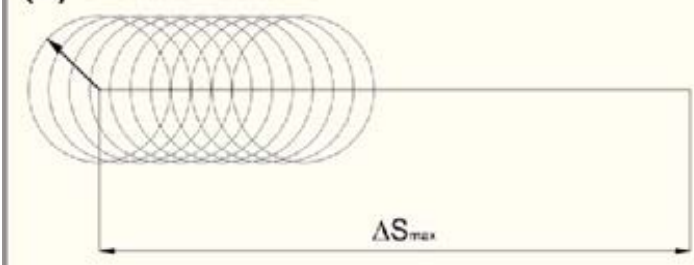

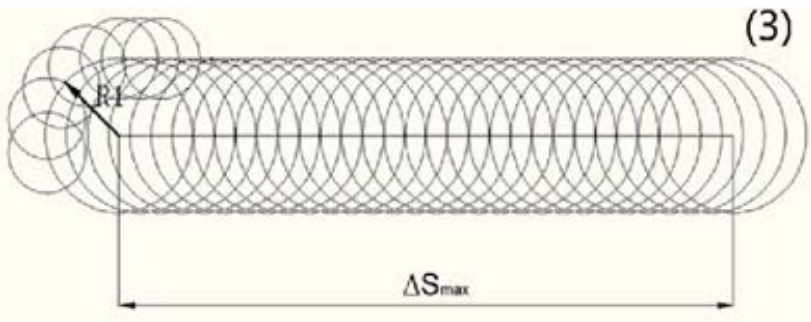

(4)

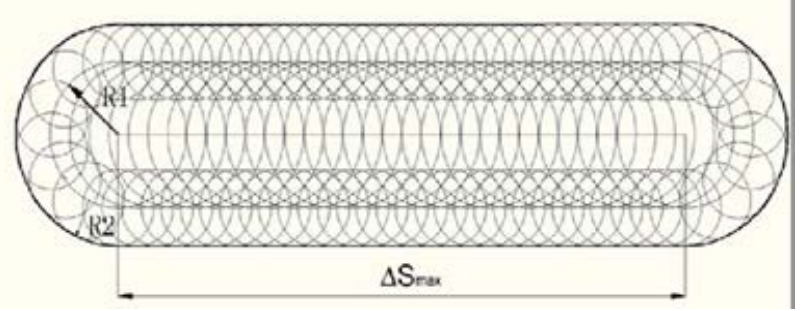

Figure 2 The forming process of search region 


\section{The Falling Model}

According to the previous hypothesis and [2] [3], after the damage of engine, the landing posture and force analysis are as Figure 3.

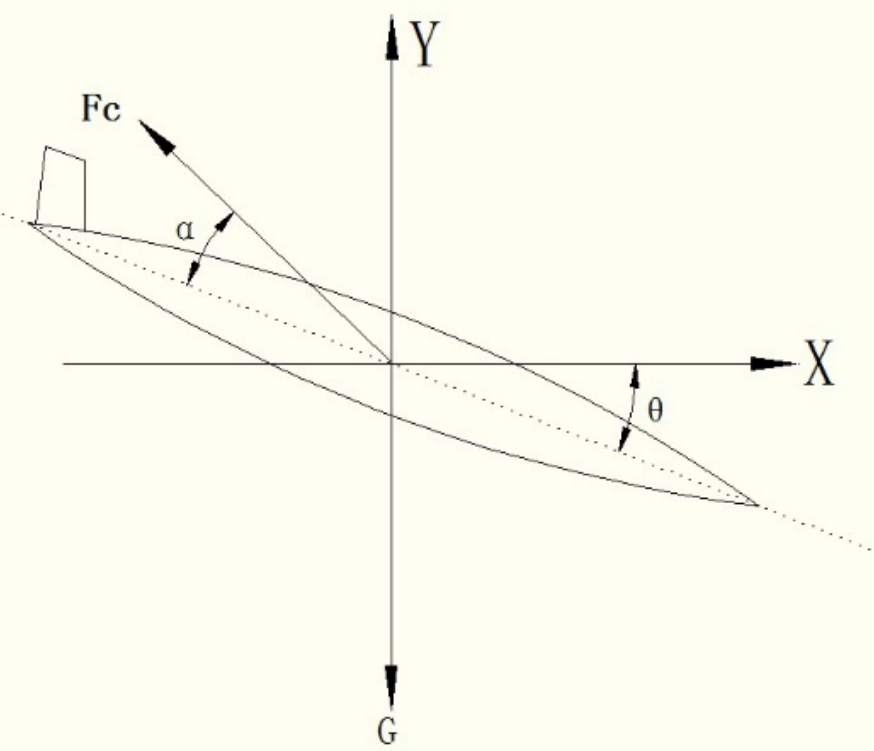

Figure 3 The force analysis when landing

Then we can get the dynamical equations along flight direction and perpendicular direction as follows.

$$
\begin{aligned}
& G \sin \theta-F_{c} \cos \alpha=\frac{G}{g} \frac{d y}{d t} \\
& G \cos \theta-F_{c} \sin \alpha=\frac{G}{g} \frac{d \theta}{d t}
\end{aligned}
$$

What's more, by geometry relationship we have:

Then, we can derive:

$$
\tan (\pi-\theta)=\frac{d y}{d x}
$$

$$
\begin{aligned}
& \sin \theta=-\frac{d y}{d x^{2}+d y^{2}} \\
& \cos \theta=\frac{d x}{d x^{2}+d y^{2}} \\
& v=\sqrt{\left(\frac{d y}{d t}\right)^{2}+\left(\frac{d x}{d t}\right)^{2}}
\end{aligned}
$$

The formula aimed to calculate the force of wind to the plane is as follows.

$$
F_{c}=\frac{1}{2} C \rho S v^{2}
$$

Where: $s$ represents the area of aerofoil; $v$ represents the real-time speed; $\rho$ represents the atmospheric density which is changes with the height and the expression is: $\rho=e^{5.25885(\operatorname{In}(288.15-0.006 h)-18.25731)} ; C$ represents a coefficient concerned with the angle $\alpha$, whose formula is

$$
\begin{aligned}
& C_{L}=0.12149-0.01714 \alpha^{2}+0.00690 \alpha^{3} \\
& \mathrm{C}=1.1488+0.11832 C_{L}{ }^{2}+0.00092 C_{L}{ }^{3}
\end{aligned}
$$

We plug $F_{c}$ intro equation(1), (2) and get:

$$
-g \frac{d y}{d x^{2}+d y^{2}}-\frac{1}{2 M} \rho \operatorname{CS}\left(d x^{2}+d y^{2}\right) \cos \alpha=\frac{\frac{d x}{d t} \frac{d^{2} x}{d t^{2}}+\frac{d y}{d t} \frac{d^{2} y}{d t^{2}}}{d x^{2}+d y^{2}}
$$




$$
g \frac{d x}{d x^{2}+d y^{2}}-\frac{1}{2 M} \rho \operatorname{CS}\left(d x^{2}+d y^{2}\right) \sin \alpha=\frac{\frac{d x}{d t} \frac{d^{2} y}{d t^{2}}-\frac{d y}{d t} \frac{d^{2} x}{d t^{2}}}{d x^{2}+d y^{2}}
$$

As to this kind differential equation of higher order, we solve them by numerical calculation means of reduced order and increment dimensional method. We set:

$$
\begin{gathered}
x_{1}=x \\
x_{2}=d x_{1} \\
x_{3}=y \\
x_{4}=d x_{3}
\end{gathered}
$$

And then we get 4 order differential equation as follows:

$$
\begin{gathered}
d x_{1}=x_{2} \\
d x_{2}=-\frac{1}{2 M} \rho S C \sqrt{d x^{2}+d y^{2}}(d y \sin \alpha+d x \cos \alpha) \\
d x_{3}=x_{4} \\
d x_{4}=-g+\frac{1}{2 M} \rho S C \sqrt{d x^{2}+d y^{2}}(d x \sin \alpha+d y \cos \alpha)
\end{gathered}
$$

Since $h, v$, headings and other parameters are changing with the specific situation and considering the fact that we adopt numerical method to solve the differential equation and use graphical method to confirm its value in the final, we can't get the specific analytic expression. So, we use the parameters of general plane to operate. Namely, we set $S$ (the reference area of aerofoil) to $75 \mathrm{~m}^{2}, \mathrm{M}$ (the weight) to $20 \mathrm{t}, \theta$ (the sliding angle) to $0, \mathrm{~V}$ (the speed) to $222 \mathrm{~m} / \mathrm{s}$ relative to the $10000 \mathrm{~m}$ airspace. There we can neglect some particular flight attitude so that to general plane, we set $\alpha$ to $\arctan (1 / 17)$.

\section{The Determination of $\boldsymbol{R}_{2}$}

Since the fuselage is heavy, we neglect the impact of marine current when the plane in the ocean. Considering the complexity of the force exerted on the plane which makes the problem intricate, we decide to solve it from a probabilistic perspective. That is to say, we limit the possible landing position in a circular region, whose possible maximum radius can be determined by the following model.

First, we assume that under the ideal condition, the plane slides along the previous direction with a rectilinear motion in the ocean. From the similarity in Figure 4.

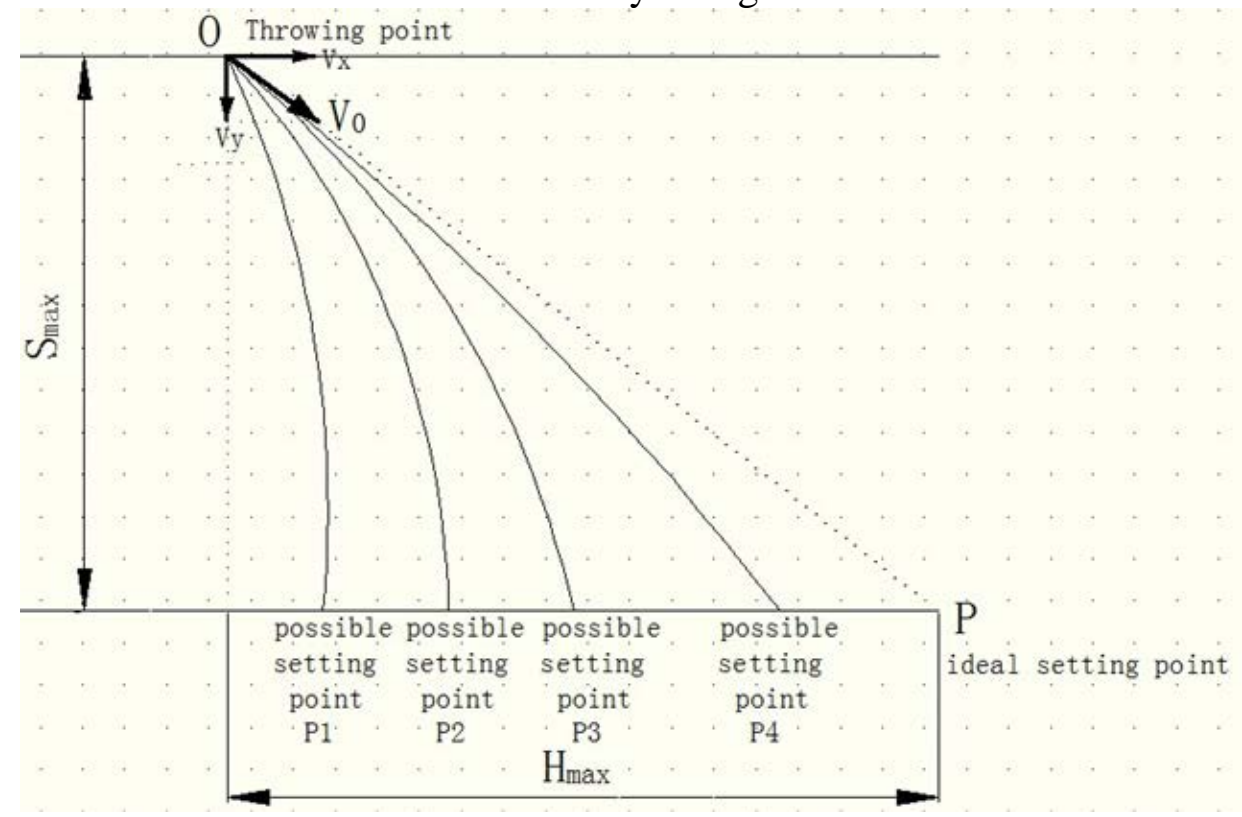

Figure 4 The setting points 
We can get the formula as follows.

$$
\frac{V_{y}}{V_{x}}=\frac{S_{\max }}{H_{\max }}
$$

Namely

$$
S_{\max }=\frac{V_{\max } H_{\max }}{V_{y}}
$$

Where: $H_{\max }$ represents the extreme depth of this sea area; $S_{\max }$ represents the maximum horizontal displacement under the ideal condition namely R2; $P_{1}$ to $P_{4}$ represents the possible landing position, Obviously, its corresponding horizontal displacement is all lesser than $S_{\max }$, which guarantees the comprehensiveness of the searching region.

Then, we divide the district getting from the first step and derive three relatively regular areas $1,2,3$. Now we select the largest region 2 to analyze. Because the area of central rectangle is too large to finish the global search in a finite time with a limited number of plane, we segment it to improve efficiency. That is to say, we first determine the minimum search region aimed to divide the region 2 into appropriate cell and then have a stochastic simulation test to predict the successfully searching probability of each region namely prior probability. Next, we determine the search path according to the corresponding probability of each region. Whenever finish searching a region, we will reallocate the probability of the rest region based on Bayes' theorem. Thus, we can determine next search cell according to the probability until searching all of the cells.

\section{Conclusion}

In this section, we define the area that 2 common search plane can finish within 3 hours by parallel search method as the minimum search region [4][5]. The abridge general view of parallel search cross section of two planes is as Figure 5.

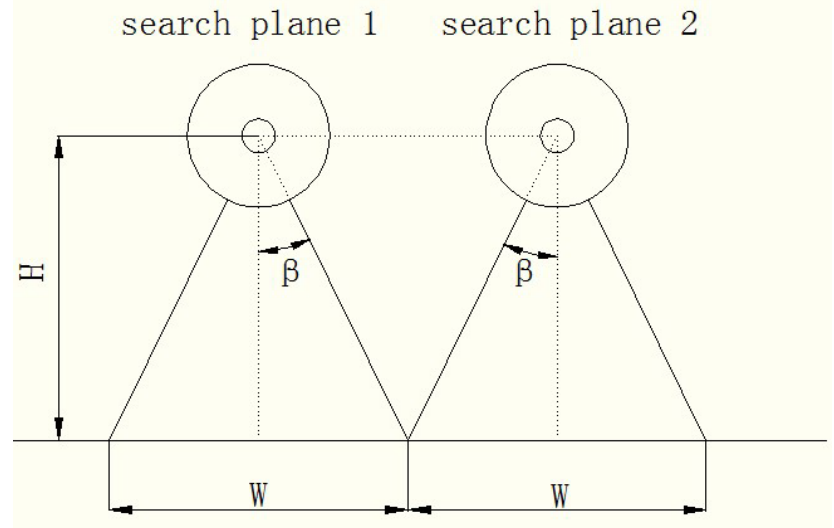

Figure 5 The search region

Then, considering that the smaller the $\mathrm{H}$ is, the nearer the search plane distant from the sea surface and the more accurate the search precision, we select the minimum altitude $H=150 \mathrm{~m}$ and $\beta$ $=45^{\circ}$. Through the geometric relationship we can get $\frac{\omega}{2 H}=\tan \beta$. So the optimum search region is $\mathrm{w}=300 \mathrm{~m}$.

The Figure 6 is a spiral search path used in micro-region, from which we can get:

$$
S_{\text {min }}=\frac{w^{2} \times 3 \times 3600}{\frac{w}{v}}
$$

There, we set v to $100 \mathrm{~km} / \mathrm{h}$, w to $300 \mathrm{~m}$ and then we have $S_{\min }=180 \mathrm{~km}^{2}$. 


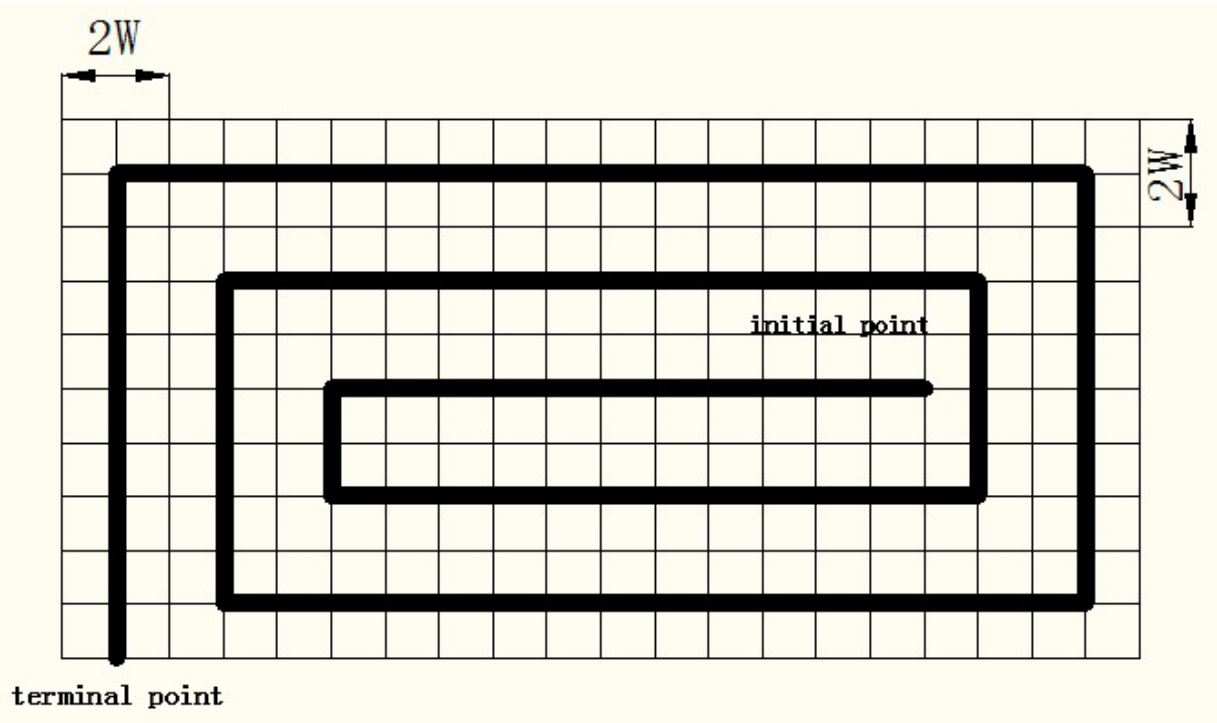

Figure 6 A spiral search path

\section{References}

[1] Bian Jiang. The research about the movement of crashed plane and black box, China,2014.

[2] Ma Peng, Feng Jia-hui, Hu Chang-yang. The calculation method on CL/CD of the wind mill generator aerofoil, China,2008.

[3] Long Di, Zhang Qi-di, The Study on the Track of Horizontal Parabola in Air Resistance, No.1,2014 (in Chinese).

[4] Liu Guang-qiang. Research on Determining Sweep Widths for Sea Searches, China,2009.

[5] Sheng-wei Xing, Research on Global Optimization Model and Simulation of Joint Aeronautical and Maritime Search, Dalian Maritime University for the degree of Doctor of Engineering, September 2012. 